\title{
Food Prohibitions and Other Traditional Practices in Pregnancy: A Qualitative Study in Western Region of Ghana
}

\author{
Patience Otoo1, Helen Habib ${ }^{2}$, Augustine Ankomah ${ }^{3 *}$ \\ ${ }^{1}$ Takoradi Hospital, Takoradi, Ghana \\ ${ }^{2}$ School of Allied Health Sciences, University of Ghana, Accra, Ghana \\ ${ }^{3}$ Department of Population, Family and Reproductive Health, School of Public Health, University of Ghana, \\ Accra, Ghana \\ Email: ${ }^{*}$ aankomah@ug.edu.gh
}

Received 5 May 2015; accepted 26 July 2015; published 30 July 2015

Copyright (C) 2015 by authors and Scientific Research Publishing Inc.

This work is licensed under the Creative Commons Attribution International License (CC BY).

http://creativecommons.org/licenses/by/4.0/

(c) (i) Open Access

\begin{abstract}
Women all over the world are confronted with many difficult choices during pregnancy and child birth. Wrong choices often result in unfavorable outcomes for expectant mothers and their babies, a situation which is common in developing countries. Cultural practices, beliefs and taboos are often implicated in determining the care received by mothers during pregnancy and child birth which is an important determinant of maternal mortality. This study explored the traditional practices associated with pregnancy and childbirth in Shama District of the Western Region, Ghana. A qualitative study consisting of six focus group discussions of between eight and ten participants per group and eight in-depth interviews were held over a period of one month. The purpose was to explore local foods that are forbidden for pregnant women and why, herbal medicine use during pregnancy and child birth and reasons for choosing home or hospital delivery. The findings show that pregnant women are forbidden from taking nutritious foods such as snails, ripe plantain, okra and many others for fear of complications during pregnancy and child birth. Herbal medicines are frequently used by pregnant women and traditional birth attendants to induce labour, augment and control bleeding during labour. Traditional beliefs and practices as well as negative attitude of health workers are found to reduce health utilization by pregnant women. Health education concerning traditional practices that are detrimental to the health of pregnant women should be emphasized during ANC visits.
\end{abstract}

\section{Keywords}

Food Taboos, Pregnancy, Traditional Practices, Food Prohibitions, Ghana

\footnotetext{
${ }^{*}$ Corresponding author.
}

How to cite this paper: Otoo, P., Habib, H. and Ankomah, A. (2015) Food Prohibitions and Other Traditional Practices in Pregnancy: A Qualitative Study in Western Region of Ghana. Advances in Reproductive Sciences, 3, 41-49. 


\section{Introduction}

Sociocultural practices and beliefs most often reflect in the attitudes of members of the community over generations [1]. Women all over the world are faced with range of choices when it comes to childbearing. Though there are direct and indirect medical causes of maternal mortality, other factors such as socio-cultural practices and poor accessibility to maternal health care could influence decisions concerning their reproductive health. These practices can therefore have either positive or negative effect on maternal health outcome [2]. In Ghana, traditional practices and religious beliefs influence pregnant women, probably because these practices and beliefs are time-tested methods which have been successfully used by generations of family and friends. A common birthing practice is for a woman to give birth completely alone which signifies her being strong, independent and a sign of fidelity to her husband [3]. This can cause delay in seeking medical care (first delay) which is one of the three delays that result in maternal death [4].

Other practices include food taboos which prevent pregnant women from eating certain foods which may be of high nutrient value such as snails and eggs. The use of herbal remedies with the intention of promoting healthy deliveries might actually be detrimental to the health of the gestational woman, as noted in Bangladesh where herbally-induced bleeding before delivery is considered culturally to be a good sign but may medically cause anaemia and haemorrhagic-related complications [5]. As a result of these practices, pregnant women may suffer from iron and protein deficiencies which could result in anemia in pregnancy which increases the risk of hemorrhagic shock and death, especially from bleeding during delivery. The survival and well-being of both the pregnant woman and her child is crucial which basically depends on the health care that the woman receives during pregnancy, at the time of delivery, and soon after delivery. Although conception and delivery are biological events, many communities attach cultural importance to the phenomenon. While many traditional societies have taboos that are applicable to all members of such societies, there are additional ones for pregnant women which are thought to protect the woman and the unborn child from evil as well as ensuring safe delivery [6] [7].

\subsection{Food Beliefs and Practices}

Several communities have food taboos for their pregnant women which in advertently deprive them of some vital nutrients [8]. Various studies have indicated that, pregnant women in different parts of the world are forced to abstain from nutritious foods [3] [9]. Taboos and misconceptions have been the custom of the people of India [8]. Among rural women of Surendranagar district, foods such as papaya, citrus foods and groundnut were avoided during pregnancy with the belief that such foods could cause abortion, placental disruption, difficult labour and many others [8]. From the fact sheet [1], the food taboos specifically for pregnant women all over communities in Africa most often exclude the consumption of nutrients which are needed by the expectant mother and fetus.

\subsection{Traditional Medicine/Herbs and Other Practices}

The use of herbs as a primary form of health care in pregnancy to facilitate childbirth is commonly practiced in many cultures worldwide. Pregnant women often use traditional medicine in order to maintain good health and reduce medical interventions [10]. In the study in Australia [10], the commonest herbs women used during pregnancy included raspberry leaf which is believed to strengthen or tone uterus ready for labour. Chamomile and ginger are used to relax the body, aid in digestion and help with nausea whilst Echinacea herbs are used to manage cold/flu as it is believed to increase immunity. In Ghana, traditional medicines used by pregnant women include Living Bitters, Yafo Pioneer Mixture, Pomaa Bitters, Yafo Tonic, and several tree bark-enemas [11]. The majority of women used these herbal medicines to treat malaria and abdominal pain, to induce smooth delivery, to keep baby healthy in uterus or to keep him kicking as well as to manage vaginal bleeding [11]. In some Asian societies, pregnant women are forbidden to sleep during the day time as it is believed to result in difficult labour, retained placenta and neonatal jaundice [12].

Maternal mortality still remains a major issue of public health concern in spite of effort put in by various countries to curtail it. However, very little is known about the traditional practices of women in Western region of Ghana especially during pregnancy and delivery. This paper catalogues some common food taboos and food prohibitions, and highlights key cultural practices and beliefs related to pregnancy and childbirth in Shama District. 


\section{Methods and Materials}

\subsection{Study Area}

The study was conducted in three sub districts in Shama District of the Western Region of Ghana, namely, Shama sub-district, Supomu-Dunkwa sub-district and Aboadze-Abuesi sub-district. The district has total population estimates of 86,983 from 2010 population census. Farming, fishing, commerce services and mining are the dominant economic activities. Seventy eight percent of the active labour force within the district are engaged in agriculture (fishing and farming), whilst industries employ 1.5\%. Services and commerce engage the rest (20.5\%) of the active labour force.

\subsection{Study Design}

This study is qualitative research consisting of focus group discussions (FGDs) and in-depth interviews (IDIs). The study consisted of FGDs among pregnant women and women who had delivered within 12 months prior to the interview and who resided in Shama district. For the IDIs, community members, opinion leaders and traditional birth attendants within the district were involved. A total of fifty-six respondents were recruited for six FGDs with between eight to ten women in each group and eight key informants were selected from all the 3 sub-district.

\subsection{Sampling Procedure}

Two communities from each of the three sub-districts (one urban and one rural) were selected for the study. Shama sub-district has only one urban community and was therefore purposively selected. The rest of the communities were categorized into rural and urban. Random selection was used to select a community each from the categories within each sub-district.

\subsection{Selection of Participants for FGDs}

At each selected community, with the help of a community based surveillance volunteer, a list of all the pregnant women and women who delivered within 12 months prior to the date of the interview was compiled. Eight to ten respondents were then randomly selected from each community list to constitute a focus group. In all, a total of six FGDs were held within all the three sub districts.

\subsection{Data Collection Procedure}

IDI's and FGDs in the form of homogenous groups were used. All the IDIs were carried out in the homes of the participants. Both the FGDs and IDIs were held at convenient spaces within the communities. The FGDs were conducted in the local language (Fante) while the IDIs were in both English and Fante. All FGDs and IDIs were audiotaped and transcribed verbatim. To add up to the transcriptions, the notes made by the researcher during the data collection were used.

\subsection{Data Analysis}

The data ensuing from the transcriptions were evaluated, coded and analyzed manually. First of all, the researchers read the transcripts several times in order to gain better understanding of the context. Major categories and major themes based on the study objectives were developed. The coded results from the FGDs were also confirmed with that of the IDIs.

\subsection{Ethical Consideration}

Written permission was obtained from the District Director of Health Services. Verbal consent was sought from community leaders and each study participant was recruited after a clear explanation about the purpose of the study and their right to withdraw their participation at any time. The participants were not paid to take part in the study but a token in the form of powder soap was given to each of them for their time. 


\section{Results}

\subsection{Socio-Demographic Characteristics of Study Participants}

A total of 64 participants were involved in both FGDs and IDIs. Fifty six respondents participated in the six FGDs with the age range of 15 to 39 years whilst eight participated (four males and four females) in the IDIs with age variation from 34 to 87 years. The average age of the respondents was 30.2 years. For the FGDs, 26 of the women were pregnant during the time of the study and 30 had delivered between five weeks and 12 months before the study. Majority $(n=54)$ of the participants were Christians. In terms of parity, only a few $(n=9)$ had not given birth before but the majority of the respondents' parity ranged from one to eight. Most of the respondents were engaged in diverse occupations (petty trading, farming, fish mongering, dressmaking and hair dressing). In addition, three teachers, four health care professionals, one banker and a retired TBA were also involved in the study. A few of the respondents were unemployed. The educational qualification of the participants included primary, junior high school/Middle School Leaving Certificate and very few had tertiary education. Table 1 shows some of the basic characteristics of the respondents.

\subsection{Food Beliefs and Practices}

Many of the respondents mentioned snails as one of the foods they believed make the baby salivate too much. Ripe plantain, due to its softness, is believed to result in lethargic and soft babies and so causes prolonged labour. Okra was perceived to cause burning sensation of the waist and painful labour while groundnut makes them sleepy during labour. Other foods avoided included Milo, malt, sweet potato, gari and mango. From the words of two participants during the FGDs, "When I was pregnant, both my grandmother and mother told me not to drink malt and Milo and also not to eat mangoes and sweet potatoes because malt and Milo will make my baby big and will let me have difficulty in labour, and with sweet potatoes, because harvesting or uprooting it is difficult, if a pregnant woman eats it, it makes her experience difficult labour" (FGD participant, 20 years). A few of the respondents who were highly educated however disagreed with the food taboos and beliefs associated with it. "As for me, I don't believe in these things because when I'm pregnant, I eat anything I feel like eating and nothing happens to either me or my baby" (FGD participant, 34 years). In addition to the food taboos, respondents were asked to provide reasons for the food women actually ate or were encouraged to eat during pregnancy and almost all talked about meat, beans, "kontomire", "agushie", "kwahunsusua" and "Keta school boys". The reasons included the following: to keep healthy during pregnancy, to make baby strong and healthy in the womb and to get enough blood. Participants also mentioned fruits, crabs and unripe plantain as good for pregnant women with the belief that fruits and crabs makes both woman and unborn baby healthy and because unripe plantain is hard, it makes the unborn baby strong and also gives the woman strength in labour.

\subsection{Traditional Medicines and Herbs}

Most of the respondents in both the FGDs and IDIs indicated that the use of herbs is a key practice both during pregnancy and child birth. Herbs such as "Setedua", "TrPntrPfo", "AkokPnyindém", "AbPwomba eguw'ekyir", and "Eban" are used for enema during pregnancy. These herbs are believed to make both mother and baby strong and healthy. "As for our traditional herbs, it's so medicinal that even if a woman would want to seek medical interventions during pregnancy, it is advisable for her to add local herbs too. I have helped several

Table 1. Characteristics of FGD participants.

\begin{tabular}{ccccc}
\hline Community & Number of Participants & Age Range & Parity & Educational Level \\
\hline Shama (urban) & 10 & $29-39$ & $3-8$ & Non, Primary, JHS, Tertiary \\
Abotaryie (rural) & 8 & $19-31$ & $1-5$ & Non, Primary, JHS \\
Inchaban (urban) & 10 & $24-37$ & $0-5$ & Non, JHS, Tertiary \\
Dwomo (rural) & 10 & $22-36$ & $0-4$ & Non, Primary, JHS \\
Supomu (rural) & 10 & $15-28$ & $0-5$ & Non, Primary, SHS \\
Bronikrom (rural) & 8 & $17-32$ &
\end{tabular}


pregnant women who bled during pregnancy. I mix herbs known as "mamafo" with the shells of an egg and "shilo" (white clay) for them to use as enema". (Retired TBA).

Most of the respondents mentioned other traditional medicine such as: "Aponsoź" which is used for enema. This is believed to remove phlegm from the mother during pregnancy so that baby does not swallow any phlegm during delivery, "We have a type of herb called Awombréduro which we normally use for enema at the eighth month with the belief of making babies strong and prepares mother for easy labour"(IDI participant, 54 years). "Nunum", 'the middle of the palm fronts', the bark of cola nut tree and "Tweta" are examples of herbs which are believed to make the baby slippery and result in easy and smooth labour. Most of the respondents confirmed using herbs to keep healthy during pregnancy and to prepare them for childbirth as shown by the following participant. " As for me, I think the use of herbs does not just protect the mother, but the baby as well because I used to suffer from fibroid a lot but orthodox medicine was not helpful so a friend thought me to be using "AkokDnyindśm mixed with white clay and another herbs called "bowin" for enema. This helped to push the fibroid aside for me to have all my five children now”. (FGD Participant, 37 years).

\subsection{Other Traditional Beliefs and Practices}

Majority of respondents mentioned other traditional cultural and behavioral practices associated with pregnancy. It was disclosed that a pregnant woman should not prepare or approach fire. It is believed to cause miscarriage or make the baby's cord long. A pregnant woman is not expected to use cloth around the chest because it would result in a baby having his/her cord around the neck. Many also mentioned that pregnant women are not to expose their chest or to help each other in carrying or offloading loads off their heads. One of the key informants gave the reason: "There are people with evil eyes so if a pregnant woman exposes her chest, they can destroy the baby or give the unborn baby a disease". "We are two pregnant women in our household. Any time one of us goes to fetch water, there will be the need to call someone to help offload because if we help ourselves, the one whose pregnancy is not old will have a miscarriage. (FGD participant, 27 years). Another mentioned: "I know also that pregnant women are not to carry heavy goods because this will lead to miscarriage”, (IDI participant, 45 years).

Most of the practices raised during the FGDs had diverse views from participants. "As for the things pregnant women are not supposed to do in our culture, they are a lot. I think some are beneficial and others are not. Our elders say that if a woman is pregnant, it is not good for someone to pass behind her or else (laughing) that person's misfortune will be upon the unborn baby. I do not know how that is possible” (FGD participant, 30 years). "My mother told me that I should not cross my legs or else my baby would delay before he/she walks and also never to stand and eat if not it will make my baby stretch at all times" (FGD participant, 19 years). "Our elders say that it is also not good for someone to kneel before a pregnant woman because this can result in a crippled baby which to me I don't believe. And with the issue that pregnant woman should not use axe to break firewood, it is believed can result in a baby having a mark on his/her head but I think it's just to prevent the mother from putting too much pressure on the baby" (FGD participant, 34 years).

\subsection{Practices during Delivery}

Participants expressed several actions they take to address prolonged labour, retained placenta and bleeding during labour. When the participants were asked what to do in case of prolonged labour, the responses were intriguing: traditional medicine, herbs to be swallowed or to be used for enema, praying to stop any spiritual attack causing the delay, or seeking medical care. "If a woman is in labour and it delays, what we normally do for her is to grind a traditional medicine called "Adaade" as enema or "Atamba" with palm kernel to be chewed to induce vomiting to ease labour" (IDI participant, 64 years). It was revealed by the majority that prolonged labour is associated with spiritual attack/curse or troublesome baby. Only a few of the respondents said it was complications in labour needing medical attention. For the management of retained placenta and bleeding at home, responses from both FGDs and IDIs indicated that it was only after the failure of traditional practices that a woman would be referred to medical interventions. The woman is given a bottle to blow air into it. This is believed to put pressure on the placenta to be expelled. Another practice is putting wooden ladle into the throat of the woman to induce vomiting or the use of herbs. Few of the respondents however argued that the use of ladle to induce vomiting is a thing of the past. "As for me when I had a retained placenta after my home delivery, the birth attendant used rags used in the kitchen to rub my back and the placenta came” (FGD participant, 19 years). 
"I delivered at home and bled afterwards. The TBA gave me "Shilo" (white clay) mixed with water to drink. After some time when it didn't stop then she asked my mother and husband to take me to the hospital" (FGD participant).

\subsection{Reasons for Home Delivery}

Findings from the study showed that usually, a combination of factors influenced the decision to have a home delivery. About half of the respondents attributed home delivery to precipitate labour, competency of birth attendants at home and successful home delivery by individuals or relatives (culture of home delivery)." I have four children and I delivered all of them at home, in my family, it is not our culture to deliver in the hospital so all my sisters delivered at home. Though we go for ANC once a while, with my last child for instance, I decided to deliver in the hospital but when I got to the clinic, the midwife told me it was not labour but the moment I went back home, the baby started coming so I delivered at home" (FGD participant, 32 years). "Reasons by participants from the FGDs were confirmed by the respondents in the IDIs. "TBAs have been with us from time immemorial and they are very good and experienced. Most often people go into labour and the baby just start coming so they (TBAs) assist them" (IDI respondent, 68 years).

Nevertheless, many of the participants were quick to highlight experiences with facility-based care that lead pregnant women to seek care from a TBA. Participants talked about too many items requested at the health facility for delivery, financial constraint and bad attitude of health workers. "They will tell us services at the health facility are free yet when you go they keep telling you to pay for this and pay for that and give you a long list on top to come with when in labour meanwhile money is hard to come by "(FGD participant, 30 years). "Most of the nurses have bad character; they treat you as if you are not a human being. I delivered my first born at Shama Health Centre and I didn't like the way I was treated so this one, I have decided to deliver at home" (FGD participant, 29 years).

Others also talked about the fact that they feel more comfortable giving birth at home than health facility due to fear of hospital environment, equipment and procedures. Also, for the fact that there is more social support at home during delivery as compared to health facility. "I am afraid to be operated on so it's a reason I had all my three children at home; as for the hospital, if the labour does not progress early they will send you to the theatre" (FGD participant, 35 years). Most of the respondents in the rural areas also attributed home delivery to long distance to health facility as compared to people in the urban area. Others were in favor of facility delivery. "I told my husband I am afraid of home delivery and will prefer to deliver at a health facility and he was ok with it" (FGD participant, 29 years).

\subsection{Perceived Health Implications Associated with Home Delivery}

The minority of women in the focus group discussions expressed several negative effects towards the health of both women and babies resulting from home delivery. They commented on the non-observance of infection prevention which exposes the woman to infection as well as the baby's cord. The issue of forcing women with obstructed labour to have a vaginal delivery was also discussed.

"As for me I do not like home delivery because the birth attendant does not wear anything on her hand so if she has any disease she can transfer it to you. Even when you cannot push, she will force you and manipulate your birth canal which can make you to lose your baby" (FGD participant, 30 years). A 31 year old participant in the focus group discussion shared her story. "It is not good to deliver at home because it has a lot of negative effects. I have three children and I had a lot of problems with the one I delivered at home. Three days after my delivery, my baby's body became hot and I realized the cord was swollen. I spent days at VRA Hospital for my baby to be treated). In spite of these responses, few of the respondents had positive comment about implications of home delivery on their health. The availability of family support was mentioned by respondents. "With home delivery, mothers and other close relatives could stay close to the women and provide psychological support as well as physical care, including back massage and gentle touching of the abdomen, soothing the woman in labour and making her feel relaxed unlike the hospital that they will put the labouring woman in a room all by herself' (IDI participant, 54 years).

\subsection{Perceived Effects of Cultural Practices}

On the question of culture and its consequences on their health, most of the respondents did not agree that, any 
of the cultural practices can have negative effect on pregnancy outcomes. They were of the view that traditional practices were meant to protect both mother and baby and to make them healthy.” These practices are what our forefathers lived on in the past and they were very healthy so I don't think it has any negative effect on our health" (IDI respondent, 53 years). These reports were confirmed during the FGDs.). "If you are pregnant and you avoid all the foods which are taboos and conform to all other practices, it's for your own good because it will protect both you (mother) and the baby but if you go contrary and you become sick or even lose your baby then you have to blame yourself' (FGD participant, 37 years).

However, many of the participants affirmed that some cultural practices have the tendency of affecting both pregnancy and childbirth negatively. "Things have changed these days so to me, I do not believe in these cultural practices. I quite remember when I was pregnant of my first child, I had abdominal pains and my mother gave me some herbs for enema and I started bleeding. Hmmm if not for God, I would have died" (FGD participant, 31 years). A few of the respondents argued that whether traditional practices have negative or positive effect depended on the baby in utero. "There are some children who do not like enema so if you are pregnant of such children and you use enema, it will worry you but if not, then there is nothing wrong with a pregnant woman using enema" (IDI participant, 68 years). "I have been pregnant for three times and for my first two children, I was managing my fever and abdominal pains with (modern) medical treatment but with the last one, it didn't like orthodox medication so it wasn't helping me when I took them. My mother advised I try herbs and it worked so that made me know that is what the baby want, (laughing)" (FGD participant). A summary of taboos, beliefs and practices that engender good health of mother and baby and those that are health impairing are depicted in Table 2.

\section{Discussion}

This study examined the taboos, beliefs and practices associated with pregnancy and childbirth among women both in the rural and urban areas in Shama district of Western region. The influence of cultural practices on the health of pregnant women and health seeking behavior were discussed. Evidence from the study showed that cultural beliefs and practices exist alongside the use of modern health care services. These beliefs and practices play a significant role in determining the food choices, activities and choice of treatment during pregnancy and delivery. Even though many of the foods mentioned are nutritious diet and facilitate good health for mother and unborn baby, most of the respondents still avoided foods which contains essential nutrients but seen as culturally forbidden in pregnancy. However, poor nutrition during pregnancy is associated with low pregnancy weight gain, high infant and maternal morbidity and mortality [13]. Studies have shown that foods such as meat, fish, grains, fruits and vegetables, when taken in adequate and varied quantities by expectant mothers is not only beneficial to the pregnant women but also to the unborn babies [8] [13].

Table 2. Beliefs and practices among women in Shama District.

\begin{tabular}{ll}
\hline \multicolumn{1}{c}{ Period } & Positive beliefs and practices \\
\hline Eregnancy & Prevention of strenuous activities \\
& Crossing of legs while sitting \\
& Negative beliefs and practices \\
& Avoidance of snails, groundnut, ripped plantain, mango, Milo \\
& The use of herbs in managing danger signs \\
& Perceiving the swelling of hands and feet, abdominal pains and profuse vomiting as normal \\
Pregnancy & Attributing edema of feet and hands to twins, baby boy, big baby. \\
& Late registration of ANC due to evil eyes/witchcraft \\
& Attributing prolonged labour to curse/spiritual forces \\
Culture of home delivery & Managing labour complications with herbs and other traditional measures \\
\hline
\end{tabular}


Respondents in the study use traditional medicine for various reasons such as keeping mother and baby healthy, treatment of various ailments during pregnancy and for management of prolonged labour, hemorrhage and retained placenta which is consistent with previous [3] [10] [11]. It is not clear what active ingredients are contained in the herbal preparations used by pregnant women in Shama. However, studies conducted on the common herbal medicines used by pregnant have been proven to produce uterotonic effect on pregnant rats [14]. This could result in poor fetal and maternal outcome due to fetal distress, uterine atony and rupture. Also, the use of herbs as the first choice of treatment during complications in labour causes delay in seeking health care and therefore can result in neonatal and maternal morbidity.

Though some of the beliefs, taboos and practices by the respondents in this study may not have scientific basis, there could be plausible beneficial effects associated with some of them in pregnancy. For example, that a pregnant woman should not tie a cloth tightly around the chest can be explained by the fact that in advanced pregnancy the gravid uterus exerts pressure on the diaphragm thereby reducing the intrathoracic pressure. Tightening a cloth on the chest will further reduce the thoracic pressure and therefore compromise respiration for both mother and baby. Most of these beliefs could therefore be precautionary [7]. Majority of the women attended ANC to confirm pregnancy and to ascertain the state of the fetus. However, some were of the opinion that early attendance at ANC, when the pregnancy is not well established (visible) will expose them to evil spirits that may harm the fetus. These findings are consistent with other studies [5] [12] [14] which have grave implication on early identification and management of at risk pregnancies. Sociocultural practices have therefore been identified as playing a significant role on the use of health care by pregnant women [15]. The findings also revealed that pregnant women with higher education are more likely to shun negative traditional practices which are in line with other studies, highlighting the important role education plays among women in choosing medical intervention over potentially harmful cultural practices. The cultural beliefs and practices however run through both rural and urban areas of the district.

Several factors influence the decision to have a home delivery. Findings from Jansen [14] indicated childbirth as being 'something natural' and not sickness and therefore no need for a pregnant woman to deliver in a health facility; she can deliver at home. Studies revealed that most pregnant women would prefer home delivery in order to get care and comfort from their families as well as to take advantage of low cost of care received from the TBAs [12] [16] [17]. Some studies, however, report that cultural practices had no negative effect on pregnancy [18]. Deliveries conducted by untrained TBAs are associated with frequent maternal mortalities due to sepsis from frequent vaginal examination with bare hands and post-partum hemorrhage resulting from inability to recognize danger signs [19]. Skilled assistance is therefore essential to safe delivery care because complications can arise at any stage of the delivery irrespective of the ANC.

\section{Study Limitation}

The study was a qualitative study and might not be a true representation of the entire district

\section{Conclusions}

This study has highlighted the importance of culture in determining the outcome of pregnancy and child birth. Negative practices such as food taboos could deprive pregnant women of good nutrition and consequently could lead to maternal anemia, low birth weight and postpartum hemorrhage. Also, the use of herbal preparations ingestion and enema to induce or augment labour can lead to unfavorable outcomes of pregnancy as some of these herbs contain uterotonics which induce uncontrolled uterine contraction during labour. Some of these beliefs and practices tend to negatively affect early utilization of the health services. Negative attitude of health workers is also noted to result in increased home delivery. Most of the women still believe in old unscientific traditions. However, the study has also identified positive cultural practices, which when promoted, will improve pregnancy outcome, thereby reducing maternal mortality.

It is recommended that health care providers should take into consideration the prevailing cultural practices in the communities in designing health education and messages that will promote the positive cultural practices and reduce the impact of negative ones among pregnant women. In this regard, nutritional value of the forbidden foods in pregnancy should be emphasized during community based maternal health education so that pregnant women will not be ignorant of the nutrition and practices that engender safe delivery. Building confidence of pregnant women about the ability of the health care system to adequately address their needs will reduce the 
negative influence of these cultural practices on pregnancy. Training on customer care and good communication skills for health care workers will reduce the negative staff attitude. This will enable pregnant women to accept health messages concerning negative cultural practices during pregnancy.

\section{References}

[1] UNCHR (2006) Harmful Traditional Practices Affecting the Health of Women and Children. Fact Sheet No. 23, UN Office of the High Commissioner for Human Rights, Geneva.

[2] Emelumadu, O.F., Ukegbu, A.U., Ezeama, N.N., Kanu, O.O., Ifeadike, C.O. and Onyeonoro, U.U. (2014) Socio-Demographic Determinants of Maternal Health-Care Service Utilization among Rural Women in Anambra State, South East Nigeria. Annals of Medical and Health Sciences Research, 4, 374-382. http://www.amhsr.org/text.asp?2014/4/3/374/133463

[3] Maimbolwa, M.C., Yamba, B., Diwan, V. and Ransjö-Arvidson, A.-B. (2003) Cultural Childbirth Practices and Beliefs in Zambia. Journal of Advanced Nursing, 43, 263-274. http://dx.doi.org/10.1046/j.1365-2648.2003.02709.x

[4] WHO (2013) World Health Report.

[5] Choudhury, N. and Ahmed, S.M. (2011) Maternal Care Practices among the Ultra Poor Households in Rural Bangladesh: A Qualitative Exploratory Study. BMC Pregnancy and Childbirth, 11, 15. http://dx.doi.org/10.1186/1471-2393-11-15

[6] Evans, E.C. (2013) A Review of Cultural Influence on Maternal Mortality in the Developing World. Midwifery, 29, 490-496. http://dx.doi.org/10.1016/j.midw.2012.04.002

[7] Liamputtong, P., Yimyam, S., Parisunyakul, S., Baosoung, C. and Sansiriphun, N. (2005) Traditional Beliefs about Pregnancy and Child Birth among Women from Chiang Mai, Northern Thailand. Midwifery, 21, 139-153. http://dx.doi.org/10.1016/j.midw.2004.05.002

[8] Parmar, A., Khanpara, H. and Kartha, G. (2013) A Study on Taboos and Misconceptions Associated with Pregnancy Among Rural Women of Surendranagar District. Age, 4, 1.

[9] Oni, O.A. and Tukur, J. (2012) Identifying Pregnant Women Who Would Adhere to Food Taboos in a Rural Community: A Community-Based Study. African Journal of Reproductive Health, 16, 67-75. http://www.ajol.info/index.php/ajrh/article/view/81290

[10] Forster, D.A., Denning, A., Wills, G., Bolger, M. and McCarthy, E. (2006) Herbal Medicine Use during Pregnancy in a Group of Australian Women. BMC Pregnancy and Childbirth, 6, 21. http://dx.doi.org/10.1186/1471-2393-6-21

[11] Omane-Adjakum (2010) Medicines Usage in Pregnancy. Kwame Nkrumah University of Science and Technology, Kumasi.

[12] Sychareun, V., Hansana, V., Somphet, V., Xayavong, S., Phengsavanh, A. and Popenoe, R. (2012) Reasons Rural Laotians Choose Home Deliveries over Delivery at Health Facilities: A Qualitative Study. BMC Pregnancy and Childbirth, 12, 86. http://dx.doi.org/10.1186/1471-2393-12-86

[13] Lartey, A. (2008) Maternal and Child Nutrition in Sub-Saharan Africa: Challenges and Interventions. Proceedings of the Nutrition Society, 67, 105-108. http://dx.doi.org/10.1017/S0029665108006083

[14] Jansen, I. (2006) Decision Making in Childbirth: The Influence of Traditional Structures in a Ghanaian Village. International Nursing Review, 53, 41-46. http://dx.doi.org/10.1111/j.1466-7657.2006.00448.x

[15] Dako-Gyeke, P., Aikins, M., Aryeetey, R., Mccough, L. and Adongo, P.B. (2013) The Influence of Socio-Cultural Interpretations of Pregnancy Threats on Health-Seeking Behavior among Pregnant Women in Urban Accra, Ghana. BMC Pregnancy and Childbirth, 13, 211. http://dx.doi.org/10.1186/1471-2393-13-211

[16] Choudhury, N., Moran, A.C., Alam, M.A., Ahsan, K.Z., Rashid, S.F. and Streatfield, P.K. (2012) Beliefs and Practices during Pregnancy and Childbirth in Urban Slums of Dhaka, Bangladesh. BMC Public Health, 12, 791. http://dx.doi.org/10.1186/1471-2458-12-791

[17] GDHS (2008) Demographic and Health Survey. Ghana.

[18] Abimbola, A.K. (2012) Socio-Cultural Factors Affecting Pregnancy Outcome among the Ogun Speaking People of Badagry area of Lagos State, Nigeria. http://ijhssnet.com/journals/Vol_2_No_4_Special_Issue_February_2012/18.pdf

[19] Prata, N., Bell, S., Holston, M. and Quaiyum, M.A. (2014) Is Attendant at Delivery Associated with the Use of Interventions to Prevent Postpartum Hemorrhage at Home Births? The Case of Bangladesh. BMC Pregnancy and Childbirth, 14, 24. http://dx.doi.org/10.1186/1471-2393-14-24 